\title{
Tunable synchrotron-like radiation from centimeter scale plasma channels
}

\author{
Min Chen ${ }^{1,2}$, Ji Luo ${ }^{1,2}$, Fei-Yu Li ${ }^{3}$, Feng Liu ${ }^{1,2}$, Zheng-Ming Sheng ${ }^{1,2,3}$ and Jie Zhang ${ }^{1,2}$
}

Synchrotron radiation (SR) sources are immensely useful tools for scientific researches and many practical applications. Currently, the state-of-the-art synchrotrons rely on conventional accelerators, where electrons are accelerated in a straight line and radiate in bending magnets or other insertion devices. However, these facilities are usually large and costly. Here, we study a compact all optical synchrotron-like radiation source based on laser-plasma acceleration either in a straight or a curved plasma channel. With the laser pulse off-axially injected, its centroid oscillates transversely in the plasma channel. This results in a wiggler motion of the whole accelerating structure and the self-trapped electrons behind the laser pulse, leading to strong synchrotron-like radiations with tunable spectra. It is further shown that a palmtop ring-shaped synchrotron is possible with current high power laser technologies. With its potential of high flexibility and tunability, such light sources once realized would find applications in wide areas and make up the shortage of large SR facilities.

Light: Science \& Applications (2016) 5, e16015; doi:10.1038/Isa.2016.15; published online 15 January 2016

Keywords: laser guiding; laser wakefield acceleration; synchrotron radiation

\section{INTRODUCTION}

Since the first observation of synchrotron radiation (SR) in a synchrotron accelerator by Pollack et al. in 1947, SR sources have shown their tremendous merits both for scientific researches and many other applications ${ }^{1-3}$. After four generations of development, SR sources have been transformed from an initial by-product to today's customized facilities ${ }^{4}$. The radiated light has also been improved significantly in terms of brightness, spectral tunability, coherency, and stability, etc ${ }^{5}$. A typical third-generation SR facility composes of an electron accelerator, a storage ring and insertion devices. The ring structures are usually hundreds of meters in diameter, which makes them always big scientific projects, costly, and not easily accessible for potential users.

On the other hand, the laser plasma-based new concept of accelerators, i.e. the laser-wakefield accelerator (LWFA), has shown rapid progress in the last 30 years ${ }^{6-8}$. In this scheme, an ultrashort intense laser pulse is used to excite a large-amplitude plasma wave with field strength of about $100 \mathrm{GV} \mathrm{m}^{-1}$. Electrons can be trapped in the plasma wave and be accelerated to $\mathrm{GeV}$ energy in a centimeter distance ${ }^{9,10}$. The new record of beam energy is $4.2 \mathrm{GeV}$, achieved at Lawrence Berkeley National Laboratory in $9 \mathrm{~cm}$ acceleration distance ${ }^{11}$. During the longitudinal acceleration, electrons in such a structure usually also undergo transverse betatron oscillations which lead to electromagnetic radiation ${ }^{12,13}$. Betatron radiation has both been studied theoretically and demonstrated experimentally ${ }^{14-17}$. A peak brightness about $10^{22}$ photons s ${ }^{-1} \mathrm{mrad}^{-2}$ $\mathrm{mm}^{-2} / 0.1 \%$ bandwidth (BW) has been observed in experiments and simulations ${ }^{18}$. Another way to use such electron beams for radiation production is by use of external insertion devices such as a magnetic wiggler or undulator ${ }^{19-21}$, or a light undulator ${ }^{22,23}$. Schlenvoigt et al. reported their experimental results of $740 \mathrm{~nm}$ radiation with a peak brilliance $6.5 \times 10^{16}$ photons $\mathrm{s}^{-1} \mathrm{mrad}^{-2} \mathrm{~mm}^{-2} / 0.1 \% \mathrm{BW}$, which is found from the interaction of a $28 \mathrm{pC}, 10$-fs-duration electron bunch with a 50 period, $1-\mathrm{m}$-long undulator ${ }^{20}$. Although the electron-betatron radiation inside a wakefield can generate high-frequency radiation, the limited tunability in the light spectrum, and brightness hinder its wide applications. The combination of LWFA with usual magnetic devices may produce high-energy photons for applications, the size of the whole facility is, however, inevitably much expanded. The design of all-optical synchrotron-like radiation devices with improved properties would be extremely attractive for broad applications.

\section{MATERIALS AND METHODS}

Here, we study new routes toward synchrotron-like radiation with laser plasma-based accelerators and undulators formed inside straight or curved plasma channels. Different from the normal betatron radiation in a wakefield, our scheme basically results from the laser centroid guiding and transverse wake structure oscillations. It gives controllable oscillation frequency, amplitude, electron beam, and radiation beam pointing. These then provide more controllability for the radiation, important for practical applications. By using multidimensional particle-in-cell (PIC) simulations, we show that both the driver laser pulse and the electron beam can be well guided over considerable distances inside the plasma channels. In a straight plasma channel, off-axis or obliquely laser injection leads to transverse oscillations of the electron beams due to the centroid oscillation of the driver laser. In a curved plasma channel, bending propagation of the driver laser and the trapped electron beams are also found. SRs are 
then generated naturally from the beam acceleration and guiding processes. Since the oscillating propagation and bending propagation of the electron beams as well as the beam energy inside the channels can be fully controlled by the laser and channel parameters, the radiation spectra can be largely tunable.

We notice that similar ideas for radiation control by using plasma channel have been proposed by some groups recently. By using a corrugated plasma channel, Pathak et al. have studied the wake oscillation inside the channel and the following tunable radiation from the oscillating electrons ${ }^{24}$. Reitsma and Jaroszynski have studied light bending in curved plasma channels. In their work, a relatively larger curvature radius of the plasma channel has been used. They also proposed to control the radiation from the wakefield-accelerated electrons by using such curved plasma channels. However, detailed studies on wakefield generation and radiation are absent ${ }^{25,26}$. In the following, we will show the possibility to achieve both undulator and bending magnet-like radiation based upon our PIC simulations.

\section{RESULTS AND DISCUSSIONS}

Laser wakefield acceleration in a straight plasma channel

To overcome the natural diffraction of a laser beam beyond the Rayleigh length $\left(Z_{\mathrm{R}}=k_{0} w_{0}^{2} / 2\right)$, a parabolic plasma channel with the density profile $n(r)=n_{0}+\Delta n r^{2} / r_{0}^{2}$ was suggested for longdistance propagation of high-power lasers ${ }^{7}$. Here, $k_{0}=2 \pi / \lambda_{0}$ is the laser wave number, $w_{0}$ is the focal spot size, $\Delta n=n\left(r_{0}\right)-n_{0}$ is the channel depth and $r_{0}$ is its width. For a matched straight plasma channel (with $\Delta n=\Delta n_{\mathrm{c}}$ and laser spot size $r_{\mathrm{s}}=r_{0}$ ), a Gaussian laser beam with $|a|^{2}=\left(a_{0} r_{0} / r_{\mathrm{s}}\right)^{2} \exp \left(-2 r^{2} / r_{\mathrm{s}}^{2}\right)$ can achieve stable propagation, where $\Delta n_{\mathrm{c}}=\left(\pi r_{\mathrm{e}} r_{0}^{2}\right)^{-1}=1.13 \times 10^{20}\left(\mathrm{~cm}^{-3}\right) / r_{0}^{2}(\mu \mathrm{m})$ with $r_{\mathrm{e}}=e^{2} / m_{\mathrm{e}} c^{2}$ the classical electron radius ${ }^{7}$. Otherwise the spot-size oscillates between $r_{\mathrm{s}}=r_{\mathrm{i}}$ and $r_{\mathrm{s}}=\left(\Delta n_{\mathrm{c}} / \Delta n\right)^{1 / 2} r_{0}^{2} / r_{\mathrm{i}}$, where $r_{\mathrm{i}}$ is the laser spot size at the entrance $(z=0)$. The oscillation period length is $\lambda_{\mathrm{os}}=\pi Z_{\mathrm{M}}\left(\Delta n_{\mathrm{c}} / \Delta n\right)^{1 / 2}$ with $Z_{\mathrm{M}}=\pi r_{0}^{2} / \lambda_{0}$ (see Figure 1a). The above analytical results are valid for the co-axis injection case. If the laser pulse is off-axis injected, the laser centroid will also oscillate transversely besides the normal self-focusing and defocusing (see Figure 1b). The oscillation period can be analyzed by comparing Figure $1 \mathrm{a}$ and $1 \mathrm{~b}$, which is found to be $\Lambda_{\mathrm{os}}=2 \lambda_{\mathrm{os}}$. We have carried out two-dimensional (2D) PIC simulations to confirm this.

The standard PIC simulations are used to study the wakefield acceleration process. Both VLPL ${ }^{27}$ and OSIRIS ${ }^{28}$ codes are used to perform the simulations and benchmarked with each other, which guarantee the correctness of the simulation results. The profile of the normalized laser electric field is given by $a=e \mathrm{E} / \mathrm{m} \omega_{0} c=a_{0} \times \exp \left(-t^{2} / L_{0}^{2}-r^{2} / w_{0}^{2}\right)$ with $a_{0}=2.0, L_{0}=9.48 T_{0}, w_{0}=10.0 \lambda_{0}$ and $\lambda_{0}=0.8 \mu \mathrm{m}$, $T_{0}=2 \pi / w_{0} \simeq 2.67 \mathrm{fs}$. The plasma density has a channel profile with $n_{0}=0.001 n_{\mathrm{c}}$, and $\Delta n=\Delta n_{\mathrm{c}}$, where $n_{\mathrm{c}} \simeq 1.7 \times 10^{21} \mathrm{~cm}^{-3}$ is the critical plasma density for the laser pulse. We have used ionization injection for controlled electron injection by setting a mixed preformed plasma ${ }^{29-33}$, composed of fully ionized He plasma (composing the plasma density of $n_{\mathrm{e}}$ ) and partially ionized nitrogen plasma with density of $n_{\mathrm{N}^{5+}}=5.0 \times 10^{-4} n_{\mathrm{c}}$. The nitrogen is located from $x=30 \lambda_{0}$ to $x=$ $50 \lambda_{0}$ with an up-ramp-plateau-down-ramp plateau $\left(5 \lambda_{0}-10 \lambda_{0}-5 \lambda_{0}\right)$ profile. This kind of distribution is widely used to obtain quasimonoenergetic electron acceleration. One notes, however, the ionization injection may be replaced by other controlled injection schemes.

In Figure 1c, we show the centroid oscillations of the laser pulse (thick solid line) and the accelerated electron beam (thin dash-dotted line) along the longitudinal acceleration distance for different channel width $r_{0}$. In the simulations, we set $\Delta n=\Delta n_{\mathrm{c}}$ and the initial off-axis displacement $Y=3 \lambda_{0}$ when the channel width $r_{0}$ is varied. From the analytical results ${ }^{7}$, the oscillation period for focusing and defocusing is $\Lambda_{\mathrm{os}}=1.58 \mathrm{~mm}$ for our simulation parameters of $r_{0}=W_{0}$. The simulation result shows $\Lambda_{\mathrm{os}}=1.56 \mathrm{~mm}$, which is close to the analytical result. For different channel widths, analytical results show $\Lambda_{\mathrm{os}} \alpha Z_{\mathrm{M}} \sqrt{\Delta n_{\mathrm{c}} / \Delta n} \alpha r_{0}^{2}$. The simulations give similar results: $\Lambda_{\text {os }}\left(r_{0}=1.2\right): \Lambda_{\text {os }}\left(r_{0}=1.0\right): \Lambda_{\text {os }}\left(r_{0}=0.8\right)=2.25: 1.56: 1.05$, which is close to $1.2^{2}: 1 \cdot 0^{2}: 0.8^{2}$. All these simulation results show that the laser centroid oscillation can be well described by the channel-guiding theory, which provides controllable beam propagation.

Besides the laser beam oscillation, Figure 1c also shows the transverse oscillation of the accelerated electron beam as a whole. Normally, an electron beam oscillates along with its driving laser beam almost synchronously except for a slight delay and deviate a little more from the channel axis than the laser beams. When the channel width reduces
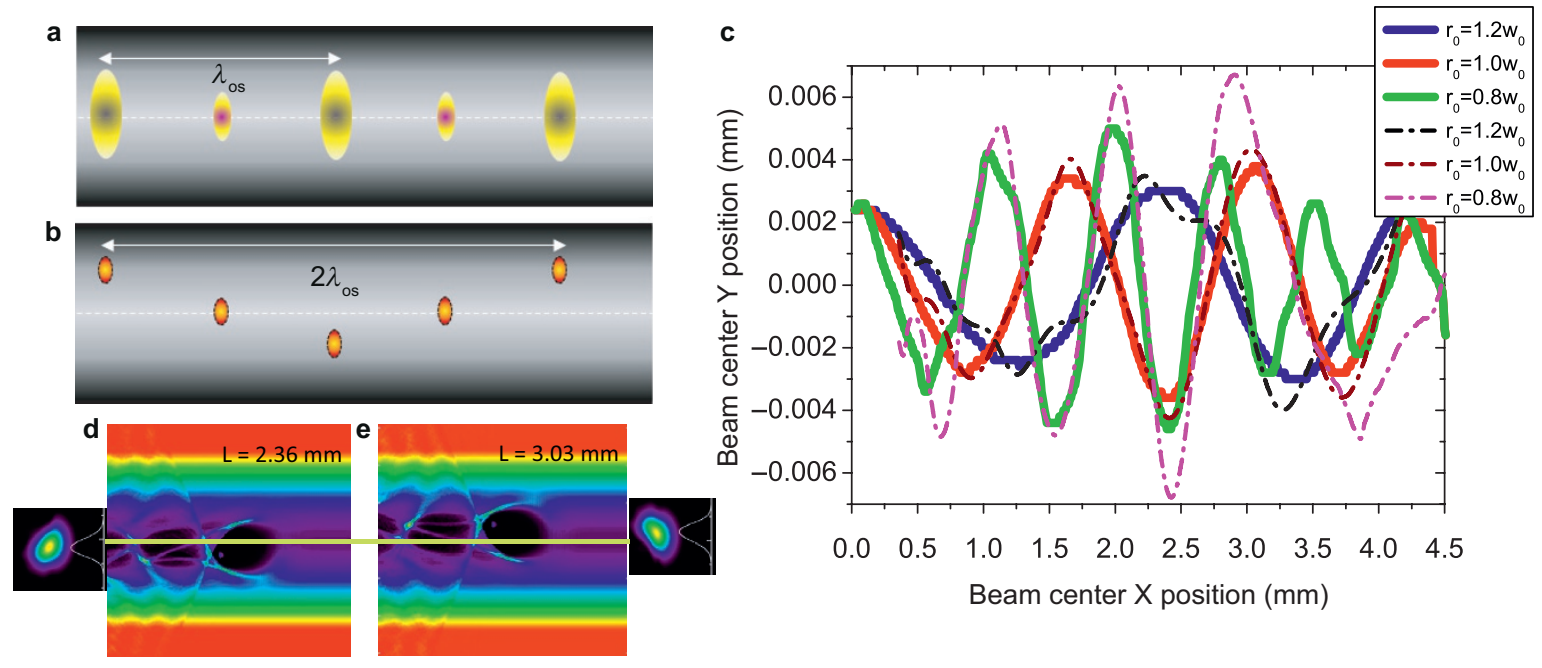

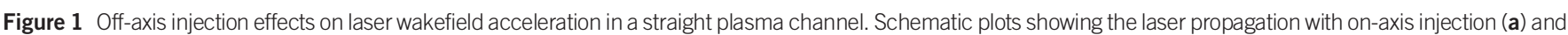

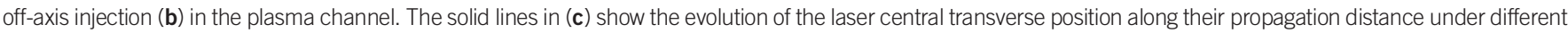

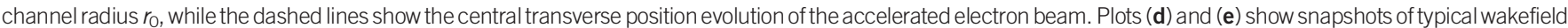
structures and accelerated beams at acceleration length of $2.36 \mathrm{~mm}$ and $3.03 \mathrm{~mm}$, respectively, along with the corresponding laser beam profiles shown at two sides. 
and the electron energy increases, this kind of synchronism is broken as shown by the green solid line and the violet dash-dotted line for the laser centroid and the electron beam, respectively. As one can see, the electron beam starts to deviate from the laser beam more and more after $x=2.75 \mathrm{~mm}$. The electron beam oscillation phase seriously falls behind the laser pulse. At $x=3.875 \mathrm{~mm}$, the slope of the trajectory of the electron beam shows somewhat discontinuity. In the simulation in this case, we find the laser pulse has been severely deformed and the electron beam directly interacts with the tail of the deformed laser pulse. This direct beam-laser interaction together with the statistical bias of the beam property due to the loss of injected electrons may be responsible for the almost discontinuous slopes of the electron beam trajectory mentioned above. Figure $1 \mathrm{~d}$ and $1 \mathrm{e}$ show snapshots of the typical acceleration structure, the electron beam and the laser beam at different time. As one can see obviously, the laser beam, the acceleration bubble, and the accelerated electrons oscillate inside the channel. This kind of beam oscillations is different from the one found by Popp et $a .^{34}$, where both the acceleration structure and the electron beam deviate from a straight line due to laser front tilting. Moreover, the electron beam cannot go back to the initial transverse position in their case.

Besides plasma channel properties, both the transverse injection deviation and laser angular pointing are critical parameters for laser propagation in the channel. In Figure 2a, three different initial off-axis positions are studied. As we can see the oscillation frequencies of the laser centroid are the same for all these cases. They are the same as the analytical results discussed before. In addition, a typical evolution of the laser-maximum intensity for the transverse off-axis of $Y=3 \lambda_{0}$ is shown by the green-dashed line. Its oscillation period is half of the laser centroid oscillation as expected according to Figure $1 \mathrm{a}$ and $1 \mathrm{~b}$. The effect of injection-pointing angles is shown in Figure 2b. The larger the injection angle, the larger oscillation amplitude of the laser beam. However, the oscillation period is independent of this parameter. It is only determined by the channel properties as shown in the analytical part. There is also some up-limit for the injection angle, beyond which the laser pulse may experience severe deformation or penetration through the channel and thus no suitable acceleration structure can be formed. For the given channel parameters in this work, the limitation is $\theta=5^{\circ}$.

In reality, laser propagation is a kind of three-dimensional (3D) phenomenon, especially the self-focusing is dimension-dependent. We have carried out 3D simulations to check the dimensional effects. Due to the limit of computational resources, we scale down the laser plasma parameters to a small acceleration structure by increasing the plasma density to $n_{0}=0.01 n_{\mathrm{c}}$ and correspondingly reduce the size of the laser pulse. The laser is initially off-axis injected into the plasma channel with $\theta=0^{\circ}$. Figure 3 shows typical snapshots of the wakefield distribution, an accelerated electron beam and longitudinal electric fields at two different acceleration distances. From the projections of the wakefield and accelerated electron beam, one can see both the laser pulse and the electron beam oscillate in the plane determined by the initial laser propagation direction and the channel axis. In the meanwhile, 2D simulations for such parameters are also carried out, which shows similar results. This benchmarks the rationality of our simulations of using 2D geometry for other cases.

In addition, there is another universal case found clearly in our 3D simulations, i.e., the centroid of a laser pulse may perform spiral motion if the initial laser propagation axis is in an arbitrary skew angle against the channel axis. This is a natural extension. The radiation by electron beams due to this motion may show unique characters.

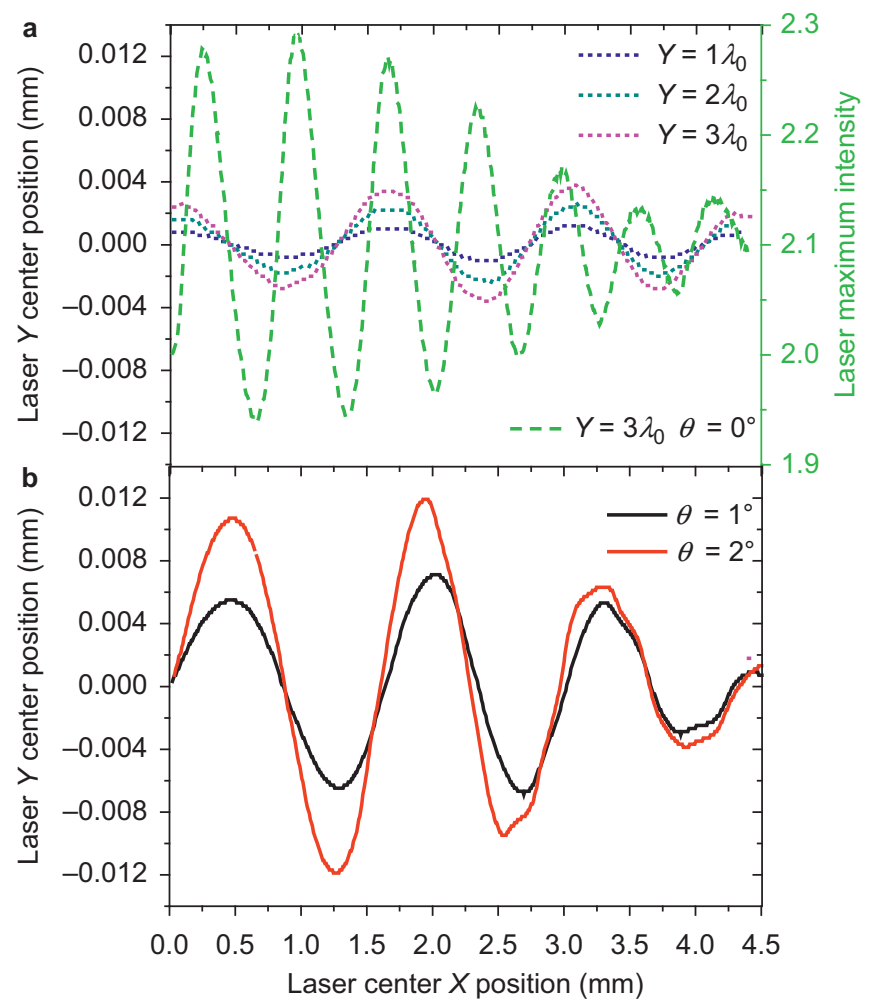

Figure 2 Laser off-axis and oblique injection effect on beam oscillation. (a) Evolution of the laser centroid for off-axis injection with different initial deviation distances $Y$. A typical laser intensity oscillation is shown by the green-dashed line. (b) Evolution of the laser centroid for oblique injection angles at $\theta=1^{\circ}$ and $\theta=2^{\circ}$ and with initial deviation distance $Y=0$. The laser and plasma density parameters are the same as before and $r_{0}=w_{0}$ for all these cases.

To avoid excessive expansion of our current paper, we leave the details in future studies.

\section{Laser wakefield acceleration in a curved plasma channel}

From the above analytical and simulation results of laser and beam oscillations, it appears possible to guide both of them even along a continuous-bending trajectory by using an initially bent plasma channel. Once realized, it can lead to controllable pointing of an electron beam and the associated radiation, which may benefit the applications of LWFA accelerated electron beam and radiation. Even a closed or open ring structure may also be possible provided the laser power is high enough and the curvature of the bent plasma channel is proper, as schematically shown in Figure 4a.

However, it is almost impossible to simulate laser guiding in a closed ring structure with the current computational ability and routine PIC codes, where the curvature of the ring is expected to be around centimeter scale or larger. Even though one cannot simulate a large range region, it is still possible to test this idea in a part of a ring structure. The plasma parameters of the arc-shaped channel are chosen according to the simulation results given above. We set the outer and inner radiuses of the ring shape plasma channel boundary to be $R_{1}=30.04 \mathrm{~mm}$ and $R_{2}=$ $29.96 \mathrm{~mm}$, respectively. The other channel density parameters and laser parameters are the same as the $2 \mathrm{D}$ simulations as before. To save the size of the simulation box, periodic boundary conditions in transverse directions are used and a moving window is used along the longitudinal direction. The box size is $80 \mu \mathrm{m} \times 192 \mu \mathrm{m}$, which is much smaller than the total simulation space $(9 \mathrm{~mm} \times 1 \mathrm{~mm})$. This is done by adjusting the 
a

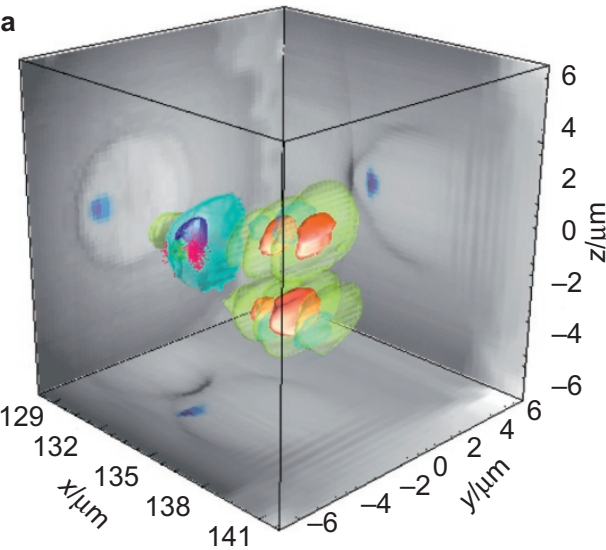

b

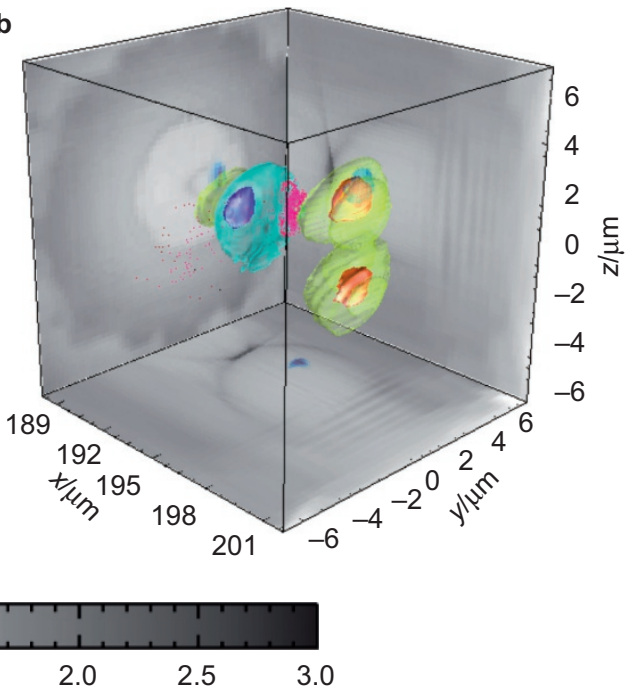

Figure 3 Typical 3D-PIC simulation results for a wakefield acceleration from an off-axis injected laser beam. Snapshots of plasma density (gray background), injected electron beam (red points) and longitudinal electric fields (blue-green-orange iso-surfaces), are shown for two different time steps. The projections on three surfaces of the cubic show the electrons oscillations along the acceleration distance. To save computational cost, laser plasma parameters are scaled down with $L_{0}=3 T_{0}$, $w_{0}=$ $4.5 \lambda_{0}, Y=3.0 \lambda_{0}, \theta=0.0^{\circ}, n_{0}=0.01 n_{\mathrm{c}}$. The nitrogen density is $n_{\mathrm{N}}=5.0 \times 10^{-4} n_{\mathrm{c}}$.

plasma channel distribution according to the box size and the transverse periodic conditions. The bent plasma channel ends at $x=7.76 \mathrm{~mm}$, after which electrons and the laser pulse propagate inside vacuum for a short distance. To avoid electron loss due to continuous bending of the wake trajectory, a 0.76-mm-long straight pre-acceleration part is added before the channel goes into the bent region. In the pre-acceleration stage electrons can be accelerated to a small distance away from the end of the bucket so there will be enough transverse potential to trap the electrons avoiding transverse losing.
Typical evolution of the laser beam centroid, the energy of beam electrons, and their average deflection angle are shown in Figure $4 \mathrm{~b}$ and $4 \mathrm{c}$. As one can see, the trajectory is generally guided by the plasma channel. From the inset of Figure $4 \mathrm{~b}$ we see the guided electron trajectory in some regions is away from the channel center. In another simulation, we find both the laser and electron beam trajectory may oscillate around the channel center as shown schematically in Figure $4 \mathrm{a}$ by the red-dashed line. Figure $4 \mathrm{c}$ shows that the electrons get continuous acceleration to $600 \mathrm{MeV}$ until arriving at the end of the
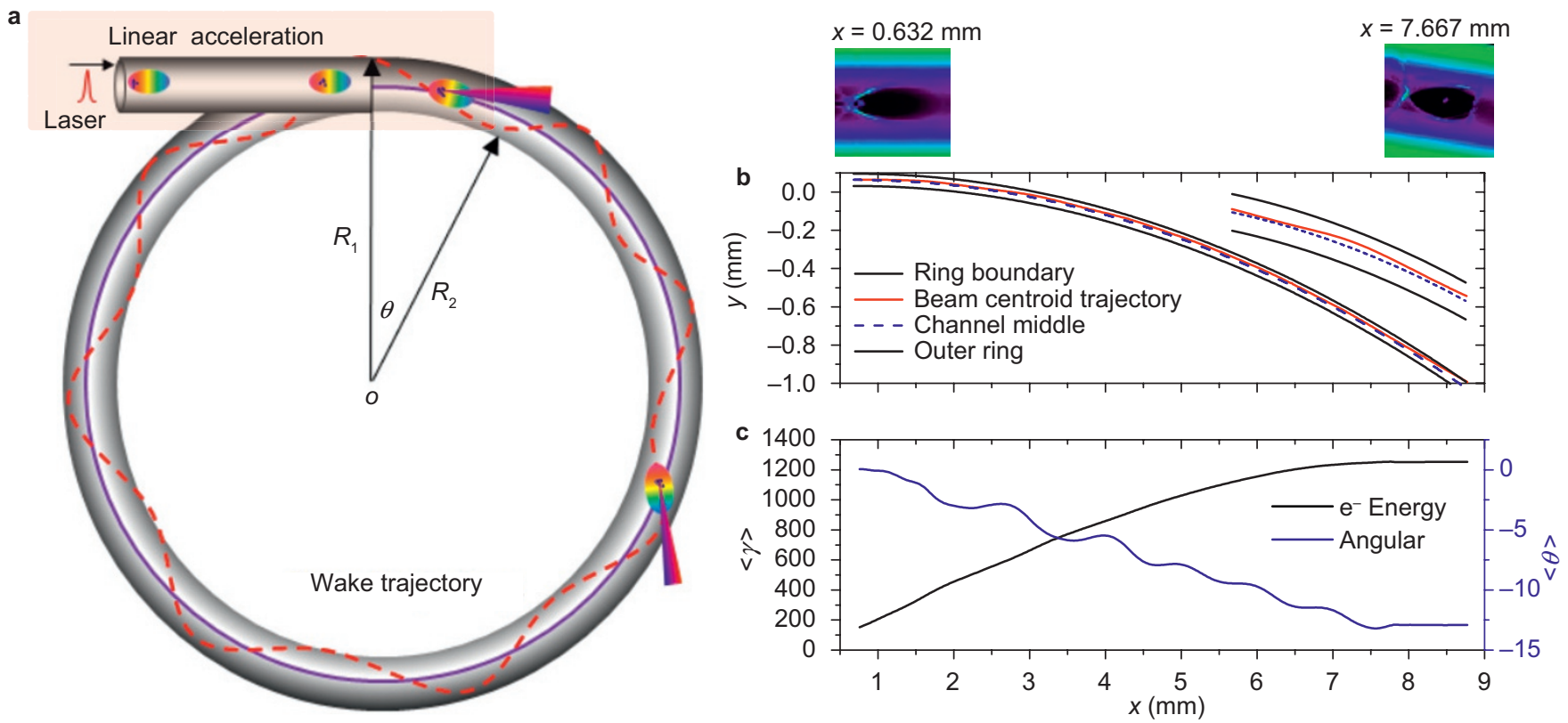

Figure 4 Electron acceleration in a curved plasma channel. (a) Schematic view of a SR ring based upon laser plasma wakefield acceleration, where the laser pulse propagates along the ring and oscillates around the channel axis with a typical trajectory shown by the red-dashed line. (b) The trajectory of the laser pulse centroid through an arc-shaped channel in $x$ - and $y$-plane from 2D-PIC simulation. In the simulation, the radius of the plasma channel is $R=\left(R_{1}+R_{2}\right) / 2=30 \mathrm{~mm}$. The top right inset of the figure shows the zoom of a part of the trajectory. One can see the laser centroid actually is only slightly deviated from the plasma center in our simulation parameters. (c) The average electron energy and deflection angle are shown. On top of (b), two snapshots of the wakefields are shown, from which one can see the wake is deflected due to the curvature of the plasma channel. 
plasma channel. The moving angle of the electron beam is shown by the blue line, which also shows an oscillating character. The final beam propagation direction is more than $12^{\circ}$ away from the initial injection direction. Two typical snapshots of acceleration structure at $x=$ $0.632 \mathrm{~mm}$ and $x=7.667 \mathrm{~mm}$ are shown on top of Figure $4 \mathrm{~b}$, which illustrate clearly the bending of the acceleration trajectory. Longer acceleration along the ring structure is possible if one uses a high energy laser pulse along with an appropriate guiding channel. Electron acceleration at the $10 \mathrm{GeV}$ level by using $10 \mathrm{~cm}$ plasma channel is well studied theoretically for BELLA acceleration in LBNL. Further acceleration length is also under discussion in many laboratories around the world. To effectively bend an electron beam, the plasma channel length can be reduced by using a smaller curvature radius. The minimum curvature depends on channel depth and laser intensity. With our simulation parameters, $R=2.4 \mathrm{~cm}$ is still possible for laser and electron beam guiding, which means the circumstance is about $15 \mathrm{~cm}$ close to the BELLA-like plasma channel length. For curved plasma channels, previous work has shown the possibility to make such channels ${ }^{35}$. In addition, since electron energy is continuously increased until dephasing happens inside the curved plasma channel, a spiral-like plasma channel with radius increasing with the polar angle may be needed to accomplish cyclotron-like radiation.

\section{Controlled synchrotron radiation in straight and curved plasma channels}

From the above studies, one can see that both in straight or curved plasma channels electrons can experience controlled transverse acceleration. Similar to the electron beams transverse acceleration in insertion devices or bending magnets in traditional storage rings, synchrotron-like radiations can be generated in such plasma channels. For incoherent radiations, the radiation intensity is proportional to the final electron beam charge. One may tune the final charge by varying the concentration of nitrogen when ionization injection is used. In our typical 2D simulations, $6 \times 10^{6} \mu \mathrm{m}^{-1} \sim 3 \times 10^{7} \mu \mathrm{m}^{-1}$ electrons are accelerated, which corresponds to about a few $p C$ electrons.

Besides the electron charge, the beam propagation trajectory is another key factor that affects the final radiation spectrum. In a straight plasma channel, electron path can be approximated by a sinusoidal curve with a period of $\Lambda_{\mathrm{os}}=2 \pi^{2} r_{0}^{2}\left(\Delta n_{\mathrm{c}} / \Delta n\right)^{1 / 2} / \lambda_{0}$, which can be tuned from hundreds of micrometers to few centimeters long. The transverse oscillation amplitude depends on the initial laser off-axis distance $(Y)$ which can be tuned up to a few micrometers. The energy of the electron beam is continuously increased during the acceleration process until reaching the dephasing point. In our typical simulations, electron energy can reach $450 \mathrm{MeV}$ after $4.5 \mathrm{~mm}$ acceleration distance. Correspondingly, the oscillation strength parameter $K=2 \pi \gamma Y / \Lambda_{\mathrm{os}}$ is widely tunable (around 0.1 to 10 ), which means the radiation can show both undulator (when $K<1$ ) or wiggler (when $K>1$ )-like spectrum. Typical radiation photon energy is, $E_{\mathrm{p}}=2 \gamma^{2} h c /\left(1+K^{2} / 2+\gamma^{2} \theta^{2}\right) \Lambda_{\mathrm{os}}$ where $h$ is the Planck constant and $\theta$ is the radiation angle.

To check the radiation, we have traced 100 electrons from the highenergy bunch in PIC simulations and input their trajectories into the VDSR code ${ }^{36}$ to calculate the radiation spectrum. The final spectrum is incoherent addition of the radiation from these 100 electrons. By selecting different end positions along the trajectories, radiations from different acceleration lengths are calculated. Typical angularintegrated radiation spectra $(\mathrm{d} l / \mathrm{d} \omega)$ from the electrons are shown in Figure 5d. Three cases are studied: (i) the plasma channel width $r_{0}=$ $w_{0}$ and the laser off-axis distance $Y=3 \lambda_{0}$ (violet and red lines); (ii) $r_{0}=w_{0}$ and $Y=2 \lambda_{0}$ (blue and black lines); (iii) $r_{0}=1.2 w_{0}$ and $Y=3 \lambda_{0}$ (green and light blue lines). Trajectories for some of the selected electrons for cases (i) and (iii) are shown in Figure 5a and 5b, respectively. They show consistency with the beam envelop evolution in Figure 1c. These oscillations are obviously different from the normal electron betatron oscillations in a wakefield as shown in Figure $5 c$, where the oscillation period increases with electron energy and the amplitude
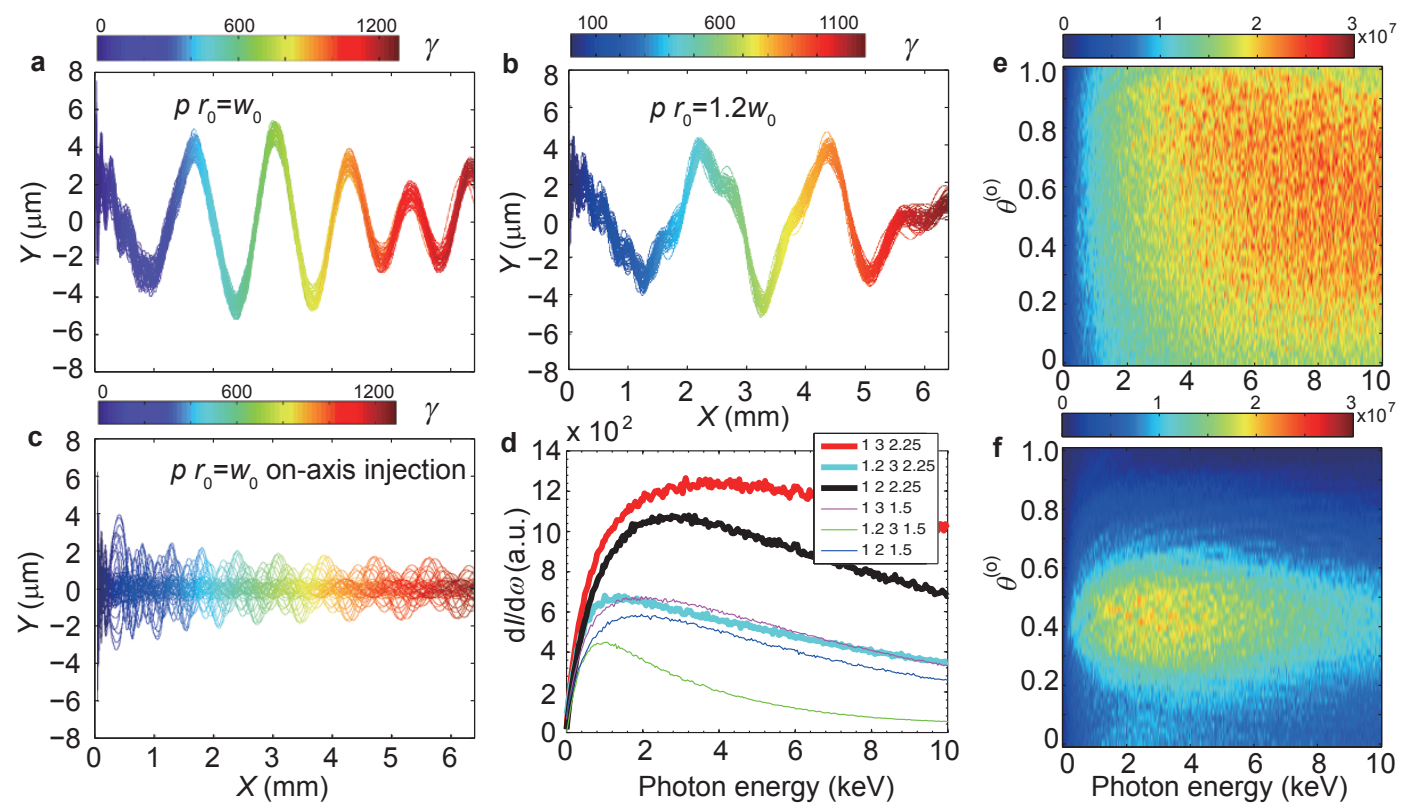

Figure 5 Typical trajectories of accelerated electrons and radiation spectra. The colors for the electron trajectories correspond to different electron energies in $(\mathbf{a}-\mathbf{c})$. The laser is p-polarized and the channel width is $r_{0}=w_{0}$ for (a) and $r_{0}=1.2 w_{0}$ for (b). (c) Normal betatron oscillation trajectories of electrons in a wakefield with on-axis laser injection. All the other simulation parameters are the same as those in Figure 1. (d) Radiation spectra for a part of the trajectories. The upright legends represent the simulation parameters: $\left[r_{0} / w_{0}, Y / \lambda_{0}, L_{a c c e}\right]$. (e and f) Radiation distribution $\mathrm{d} / / \mathrm{d} \omega \mathrm{d} \Omega$ for case (i and iii), respectively. The acceleration distance here is $L_{\text {acce }}=3 \mathrm{~mm}$. 
decreases, and the phase mixing of the electrons' motion happens at the beginning. In our scheme, electrons show small irregular oscillations overlapping on regular oscillations at the beginning of the acceleration $(x<1.588 \mathrm{~mm})$ as found in the electron trajectories. To avoid the uncertainty of trajectory selection at the beginning, the radiation calculation position begins at $x=1.588 \mathrm{~mm}$. Low-frequency radiations before this position have been omitted. Radiation spectra after two different acceleration distances are shown. The green, violet, and blue lines correspond to an acceleration distance of $L_{\text {acce }}=1.5 \mathrm{~mm}$. Light blue, red, and black lines correspond to the acceleration distance of $L_{\text {acce }}=2.25 \mathrm{~mm}$. As one can see the radiation spectra show wide BW. The peak positions of spectra for the long acceleration show obvious blue shift due to higher electron energy along the acceleration (see the color variation along each trajectory). Comparing cases (i) and (iii), one can see that a larger channel width introduces a larger oscillation period of the electron beam $\left(\Lambda_{\mathrm{os}}\right)$ and a smaller photon energy. For our simulation parameters, the peak radiation frequency locates between $0.895 \mathrm{keV}$ and $3.88 \mathrm{keV}$. The high-energy tail extends to tens of $\mathrm{keV}$, which covers the ultraviolet to hard $\mathrm{x}$-ray regime. Figure 5e and $5 \mathrm{f}$ show angular-resolved spectra for cases (i) and (iii), respectively. As one can see that besides frequency shifting, the radiation angular distribution also varies significantly with the laser plasma parameters. This provides the flexibility for radiation pointing. We notice recently, by applying the similar idea and using an externalinjected electron beams, narrow radiation spectra can be obtained if the electrons are injected into the appropriate phase of such plasma undulators ${ }^{37}$.

In a bent plasma channel, the curvature of the electron trajectories basically depends on the curvature of the plasma channel. Usually, the curvature radius $R$ is in few centimeter scale. Different from the normal radiation in a storage ring, in our case the electrons are accelerated continuously when they radiate. The radiation spectrum is more like the continuous radiation spectrum from a single-bending magnet without harmonic characters. The critical radiation frequency is about $\omega_{\mathrm{c}} \sim \gamma^{3} c / R$ for the case in Figure 4, there are $\gamma \simeq 1200$ and $R=3 \mathrm{~cm}$ at the end of the plasma channel. This corresponds to the critical photon energy of $11.3 \mathrm{keV}$.

\section{CONCLUSIONS}

In summary, a scheme of compact all-optical SR is proposed based upon LWFA by use of different plasma channels. For a straight plasma channel, electron beam oscillation and subsequent radiation are realized by the use of off-axis injection or oblique injection of laser pulses. On this basis, we show that bending laser propagation and electron acceleration inside a curved plasma channel are possible, which lead to a new type of compact SR in LWFA. In our scheme both transverse and longitudinal acceleration comes from the laser wakefield, without any external acceleration fields and bending magnets. Note that the two kinds of beam lines suggested with straight plasma channels and curved plasma channels are similar to conventional linear undulator radiation and storage ring radiation. The acceleration and radiation parts of this kind of devices can be made within centimeter size and thereby be constructed with much reduced cost. Although the quality of such light sources may not be comparable immediately with existing SR sources, it shows unique advantages for wide applications. Even though the present work has demonstrated the principle concept of the new type of SRs, it is obvious that there are still high potential and need to improve the quality of such light sources with detailed design for LWFA and channel guiding. More systematic scaling studies including both acceleration and radiation parts are still needed to make it to be a real auxiliary device to the current SR sources.

It deserves to point out that our channel-guiding studies for both laser and electron beams may also benefit other radiation schemes. By using a plasma channel, all optical Thomson or Compton scattering may be realized more easily ${ }^{38}$. Two lasers can be incident into an endtailored plasma channel from two ends separately and then guided. The intense short pulse drives a wakefield, accelerating electrons behind. The other low-intensity long laser pulse oppositely propagates and interacts with the accelerated electron beam and makes Thomson scattering. By using this method, the difficulty of laser and electron beam overlapping in space and time could be significantly reduced since both of them can be automatically guided by the same plasma channel. Detailed studies on this topic still need to be carried out. The curved channel guiding may also be helpful for staged wakefield acceleration since electrons may always be accelerated within a curved plasma channel and driver lasers with different propagation directions can be used for different acceleration stages, which avoids the usual electron beam emittance increasing between two plasma channels leading to low coupling efficiency from one stage to another ${ }^{39}$.

\section{AUTHORS' CONTRIBUTIONS}

M C conceived the idea; M C, F Y L, F L, J L, and Z M S performed the PIC simulations and radiation calculations; M C, Z M S, F Y L, and J Z analyzed data and wrote the paper. All authors commented on the manuscript and agreed on the contents.

\section{CONFLICTS OF INTEREST}

The authors declare no competing financial interests.

\section{ACKNOWLEDGEMENTS}

This work is supported in part by the National Basic Research Program of China (Grant No 2013CBA01504), Major State Basic Research Development Program of China (2015CB859700), and the National Science Foundation of China (Grant Nos. 11421064, 11374209, 11374210). M.C. appreciates supports from National 1000 Young Talent Program. Z.M.S. acknowledges the support of the University of Strathclyde and helpful comments from Prof. D. A. Jaroszynski. The authors would like to acknowledge the OSIRIS Consortium for the use of OSIRIS and the visXD framework. Simulations were performed on the П Supercomputer at Shanghai Jiao Tong University.

Huxley HE, Faruqi AR, Bordas J, Koch MHJ, Milch JR. The use of synchrotron radiation in time-resolved X-ray diffraction studies of myosin layer-line reflections during muscle contraction. Nature 1980; 284: 140-143.

2 Chen L, Dürr KL, Gouaux E. X-ray structures of AMPA receptor-cone snail toxin complexes illuminate activation mechanism. Science 2014; 345: 1021-1026.

3 Lee CH, Lü W, Michel JC, Goehring A, Du J et al. NMDA receptor structures reveal subunit arrangement and pore architecture. Nature 2014; 511: 191-197.

4 Schoenlein RW, Chattopadhyay S, Chong HH, Glover TE, Heimann PA et al. Generation of femtosecond pulses of synchrotron radiation. Science 2000; 287: 2237-2240.

5 Light sources of the World. http://www.lightsources.org/regions.

6 Tajima T, Dawson JM. Laser electron accelerator. Phys Rev Lett 1979; 43: 267.

7 Esarey E, Schroeder CB, Leemans WP. Physics of Laser-driven plasma-based electron accelerators. Rev Mod Phys 2009; 81: 1229-1285.

8 Hooker SM. Developments in laser-driven plasma accelerators. Nat Photonics 2013; 7: 775-782.

9 Leemans WP, Nagler B, Gonsalves AJ, Tóth C, Nakamura K et al. GeV electron beams from a centimetre-scale accelerator. Nat Phys 2006; 2: 696-699.

10 Wang XM, Zgadzaj R, Fazel N, Li ZY, Yi SA et al. Quasi-monoenergetic laser-plasma acceleration of electrons to $2 \mathrm{GeV}$. Nat Commun 2013; 4: 1988.

11 Leemans WP, Gonsalves AJ, Mao HS, Nakamura K, Benedetti C et al. Multi-Gev electron beams from capillary-discharge-guided subpetawatt laser pulses in the self-trapping regime. Phys Rev Lett 2014; 113: 245002.

12 Corde S, Phuoc KT, Beck A, Lambert G, Fitour R et al. Femtosecond x rays from laserplasma accelerators. Rev Mod Phys 2013; 85: 1 
13 Esarey $E$, Shadwick BA, Catravas $P$, Leemans WP. Synchrotron radiation from electron beams in plasma-focusing channels. Phys Rev E2002; 65: 056505.

14 Schnell M, Sävert A, Uschmann I, Reuter M, Nicolai M et al. Optical control of hard X-ray polarization by electron injection in a laser wakefield accelerator. Nat Commun 2013; 4: 2421

15 Kiselev S, Pukhov A, Kostyukov I. X-ray generation in strongly nonlinear plasma waves. Phys Rev Lett2004. 93: 135004.

16 Rousse A, Phuoc KT, Shah R, Pukhov A, Lefebvre E et al. Production of a keV x-ray beam from synchrotron radiation in relativistic laser-plasma interaction. Phys Rev Lett 2004; 93: 135005.

17 Kneip S, Nagel SR, Bellei C, Bourgeois N, Dangor AE et al. Observation of synchrotron radiation from electrons accelerated in a petawatt-laser-generated plasma cavity. Phys Rev Lett 2008; 100: 105006

18 Kneip S, McGuffey C, Martins JL, Martins SF, Bellei C et al. Bright spatially coherent synchrotron X-rays from a table-top source. Nat Phys 2010; 6: 980-983.

19 Nakajima K. Towards a table-top free-electron laser. Nat Phys 2008; 4: 92-93.

20 Schlenvoigt HP, Haupt K, Debus A, Budde F, Jäckel O et al. A compact synchrotron radiation source driven by a laser-plasma wakefield accelerator. Nat Phys 2008; 4: 130-133.

21 Fuchs M, Weingartner R, Popp A, Major Z, Becker S et al. Laser-driven soft-X-ray undulator source. Nat Phys 2009; 5: 826-829.

22 Phuoc KT, Corde S, Thaury C, Malka V, Tafzi A et al. All-optical Compton gamma-ray source. Nat Photonics 2012; 6: 308-311.

23 Cipiccia S, Islam MR, Ersfeld B, Shanks RP, Brunetti E et al. Gamma-rays from harmonically resonant betatron oscillations in a plasma wake. Nat Phys 2011; 7: 867-871.

24 Pathak VB, Martins JL, Vieira J, Fonseca RA, Silva LO. Laser wakefield acceleration in corrugated plasma channel. Proceedings of the 41st EPS Conference on Plasma Physics; 23-27 June 2014; Berlin, Germany, 2014.

25 Reitsma A, Jaroszynski D. Propagation of a short intense laser pulse in a curve plasma channel. IEEE Trans Plasma Sci 2008; 36: 1738-1745.

26 Reitsma AJW, Jaroszynski DA. Light bending in curved plasma channels. Central Laser Facility Annual Report 2006/2007, pp. 83-86.

27 Pukhov A. Three-dimensional electromagnetic relativistic particle-in-cell code VLPL (virtual laser plasma lab). J Plasma Phys 1999; 61: 425-433.

28 Fonseca RA, Silva LO, Tsung FS, Decyk VK, Lu W et al. OSIRIS: a three-dimensional, fully relativistic particle in cell code for modeling plasma based accelerators. In: Sloot PMA, Hoekstra AG, Tan CJK, Dongarra JJ, editors. Computational Science: Lecture Notes in Computer Science. Berlin Heidelberg: Springer; 2002; 2331: 342-351.
29 Chen M, Sheng ZM, Ma YY, Zhang J. Electron injection and trapping in a laser wakefield by field ionization to high-charge states of gases. J Appl Phys 2006; 99: 056109.

30 Chen M, Esarey E, Schroeder CB, Geddes CGR, Leemans WP. Theory of ionizationinduced trapping in laser-plasma accelerators. Phys Plasmas 2012; 19: 033101.

31 Liu JS, Xia CQ, Wang WT, Lu HY, Wang C et al. All-optical cascaded laser wakefield accelerator using ionization-induced injection. Phys Rev Lett 2011; 107: 035001.

32 Pak A, Marsh KA, Martins SF, Lu W, Mori WB et al. Injection and trapping of tunnelionized electrons into laser-produced wakes. Phys Rev Lett 2010; 104: 025003.

33 Zeng M, Chen M, Yu LL, Mori WB, Sheng ZM et al. Multi-chromatic narrow-energyspread electron bunches from laser-wakefield acceleration with dual-color lasers. Phys Rev Lett 2015; 114: 084801.

34 Popp A, Vieira J, Osterhoff J, Major Z, Hörlein R et al. All-optical steering of laserwakefield-accelerated electron beams. Phys Rev Lett 2010; 105: 215001.

35 Ehrlich Y, Cohen C, Zigler A, Krall J, Sprangle P et al. Guiding of high intensity laser pulses in straight and curved plasma channel experiments. Phys Rev Lett 1996; 77: 4186-4189.

36 Chen M, Esarey E, Geddes CGR, Schroeder CB, Plateau GR et al. Modeling classical and quantum radiation from laser-plasma accelerators. Phys Rev ST Accel Beams 2013; 16: 030701.

37 Rykovanov SG, Schroeder CB, Esarey E, Geddes CGR, Leemans WP. Plasma undulator based on laser excitation of wakefields in a plasma channel. Phys Rev Lett 2015; 14: 145003.

38 Chen S, Powers ND, Ghebregziabher I, Maharjan CM, Liu C et al. MeV-energy X rays from inverse compton scattering with laser-wakefield acelerated electrons. Phys Rev Lett 2013; 110: 155003 .

39 Thaury C, Guillaume E, Döpp A, Lehe R, Lifschitz A et al. Demonstration of relativistic electron beam focusing by a laser-plasma lens. Nat Commun 2015; 6: 6860 .

(c) (i) (2) This work is licensed under a Creative Commons Attribution-

BY NC SA NonCommercial-ShareAlike 4.0 Unported License. The images or other third party material in this article are included in the article's Creative Commons license, unless indicated otherwise in the credit line; if the material is not included under the Creative Commons license, users will need to obtain permission from the license holder to reproduce the material. To view a copy of this license, visit http://creativecommons.org/licenses/by-nc-sa/4.0/ 